coming life threatening when treating patients with an abnormal perioperative course.

\section{References}

1. Lin TY, Chiu KM, Wang MJ, Chu SH. Carbon dioxide embolism during endoscopic saphenous vein harvesting in coronary artery bypass surgery. J Thorac Cardiovasc Surg. 2003;126:2011-5.

2. Banks TA, Manetta F, Glick M, Graver LM. Carbon dioxide embolism during minimally invasive vein harvesting. Ann Thorac Surg. 2002;73: 296-7.
3. Bitondo JM, Daggett WM, Torchiana DF, Akins CW, Hilgenberg AD, Vlahakes GJ, et al. Endoscopic versus open saphenous vein harvest: a comparison of postoperative wound complications. Ann Thorac Surg. 2002;73:523-8.

4. Davis Z, Jacobs HK, Zhang M, Thomas C, Castellanos Y. Endoscopic vein harvest for coronary artery bypass grafting: technique and outcomes. J Thorac Cardiovasc Surg. 1998;116:228-35.

5. Chavanon O, Tremblay I, Delay D, Bouveret A, Blain R, Perrault LP. Carbon dioxide embolism during endoscopic saphenectomy for coronary artery bypass surgery. J Thorac Cardiovasc Surg. 1999;118: $557-8$.

\title{
Unusual chronic pacemaker infection by Mycobacterium tuberculosis in a pediatric patient
}

Thomas Hellwig, MD, ${ }^{a}$ Phalla Ou, MD, ${ }^{a}$ Catherine Offredo, MD, ${ }^{b}$ Dorothée Stephany, MD, ${ }^{c}$ Damien Bonnet, MD, $\mathrm{PhD},{ }^{a}$ and Daniel Sidi, MD, PhD, ${ }^{\mathrm{a}}$ Paris, France

$\mathrm{P}$

acemaker infection with Mycobacterium tuberculosis has not been reported previously. We describe a case of pacemaker infection with M tuberculosis in an 8-year-old patient in a country in which tuberculosis is not endemic.

\section{Clinical Summary}

An 8-year-old patient had been followed up at our institution for a congenital cardiac malformation with a single-ventricle physiology. After multiple cardiac surgical interventions, an epicardiac pacemaker was implanted during cavopulmonary arterial anastomosis surgery for a complete atrioventricular block when the patient was 6 years old.

After a symptom-free interval of 11 months, the patient was seen with a subcutaneous abscess of the pacemaker site. The pacemaker was surgically removed, although only three of the four leads could be removed because one had integrated entirely into the myocardium. Bacteriologic cultures of the surgically removed material remained sterile. After 5 weeks of probabilistic intravenous antibiotic therapy, a new epicardiac pacemaker was implanted at a different site.

Six months later, the patient was seen with decreased general health, moderate fever, and inflammatory syndrome. A battery of blood cultures yielded negative results. Echocardiography did not find any evidence of endocarditis. While awaiting more specialized inves-

From Cardiologie Pédiatrique, ${ }^{\text {a }}$ Laboratoire de Bactériologie-VirologieParasitologie, ${ }^{\mathrm{b}}$ and Service des Maladies Infectieuses et Tropicales, ${ }^{\mathrm{c}}$ Hôpital Necker-Enfants Malades, Paris, France.

Received for publication April 4, 2004; accepted for publication April 21, 2004.

Address for reprints: Phalla Ou, MD, Service de Cardiologie Pédiatrique, Hôpital Necker-Enfants Malades, 149 rue de Sèvres 75743, Paris Cedex 15, France (E-mail: phalla.ou@nck.ap-hop-paris.fr).

J Thorac Cardiovasc Surg 2005;130:937-8

$0022-5223 / \$ 30.00$

Copyright $\odot 2005$ by The American Association for Thoracic Surgery doi:10.1016/j.jtcvs.2005.04.016

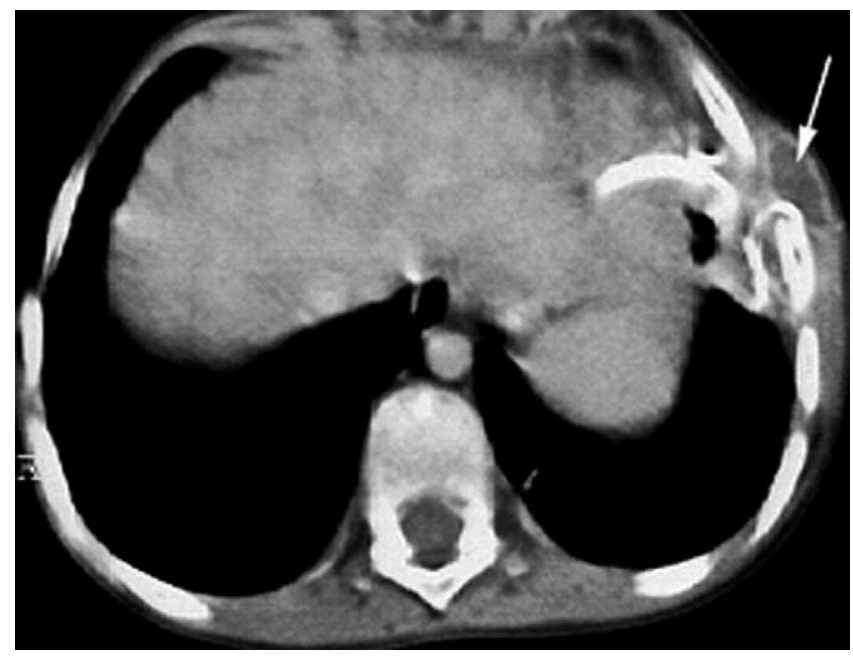

Figure 1. Thoracic computed tomography. Note soft-tissue abscess (arrow) containing pacing wires and inflammatory process following wire pathway.

tigations, we observed a subcutaneous lump above the new pacemaker site. The abdominothoracic computed tomographic scan (Figure 1) showed the typical aspect of a soft-tissue abscess, containing the pacing wires and accompanied by an inflammation following the wire pathway. After complete drainage and surgical ablation of the pacemaker, acid-alcohol-resistant bacilli were found on ZiehlNeelsen stain, and the diagnosis of $M$ tuberculosis was confirmed by IS6110-based polymerase chain reaction assay and by culture of the surgically drained fluid and pacemaker leads. The Mantoux test showed a positive reaction in the bacille Calmette-Guérin-vaccinated patient, who had never been in a country in which tuberculosis was endemic. Results of Mantoux tests and chest radiographs of the 5 
members of the patient's direct household who were of North African origin but resident in France remained negative. Microbiologic investigations of a potential origin of the infection in our institution also yielded negative results.

After this unusual pacemaker infection, we investigated further the hypothesis of a relatively immune-depressed background in this patient, who had undergone partial resection of the thymus during cardiac surgery in the neonatal period. The lymphocyte immunophenotype showed a normal profile, and an analysis of interleukin 2 and interferon gamma did not reveal an immune-depressed context. $\mathrm{Hu}-$ man immunodeficiency virus serologic results remained negative. Extensive investigation did not reveal other infected sites.

A quadruple treatment with rifampin (INN: rifampicin), isoniazid, ethambutol, and pyrazinamide for 2 months was followed by double therapy (isoniazid and rifampin) for another 7 months. The strain was susceptible to the 4 antituberculosis drugs. The patient has had good clinical progress with the antituberculosis treatment, but the implantation of a new pacemaker was delayed for several months to prevent recurrence of the infection.

\section{Discussion}

Recurrent and chronic infection of a pacemaker site is a frequent complication once a first infection has occurred. The incidence is higher in the pediatric population and increases with the number of changes of the pacemaker. ${ }^{1}$ Complete surgical removal of the pacing material is therefore considered to be the most effective treatment. ${ }^{1-3}$

Identification of the infectious agent is crucial to target the antibiotic treatment. In $40 \%$ of infected patients, staphylococci are detected in bacteriologic cultures, whereas in $40 \%$ of surgically removed pacing leads, no infectious agent is found. ${ }^{2,3}$ Pacemaker infections by mycobacterial species other than $M$ tuberculosis are rare but well documented. ${ }^{4,5}$ To our knowledge, this is the first case of a pacemaker infection with $M$ tuberculosis. The source may have been among the visitors from the North African origin of the family, where tuberculosis is endemic, but we were unable to investigate. Our case report emphasizes the importance of considering mycobacteria in pacemaker infections, particularly when Gram staining or standard cultures yield negative results.

However rare, pacemaker infection with $M$ tuberculosis must be considered in a patient with a chronic infection, particularly when Gram stain or standard culture results are negative. This is especially true for patients with a family background from a high-burden country.

\section{References}

1. Cohen MI, Bush DM, Gaynor JW, Vetter VL, Tanel RE, Rhodes LA. Pediatric pacemaker infections: twenty years of experience. $J$ Thorac Cardiovasc Surg. 2002;124:821-7.

2. Darouiche RO. Treatment of infections associated with surgical implants. N Engl J Med. 2004;350:1422-9.

3. Baddour LM, Bettmann MA, Bolger AF, Epstein AE, Ferrieri P, Gerber MA, et al. Nonvalvular cardiovascular device-related infections. Circulation. 2003;108:2015-31.

4. Kessler AT, Kourtis AP. Mycobacterium abscessus as a cause of pacemaker infection. Med Sci Monit. 2004;10:CS60-2.

5. Sharma S, Tleyjeh IM, Espinosa RE, Costello BA, Baddour LM. Pacemaker infection due to Mycobacterium fortuitum. Scand J Infect Dis. 2005;37:66-7.

\title{
Intractable postoperative internal thoracic artery spasm managed with angiographic intraluminal papaverine
}

\author{
Lucas H. A. Sanders, FCS(SA), MD, and Mark A. J. Newman, FRACS, Perth, Australia
}

pasm of the left internal thoracic artery (LITA) and native coronary artery in the immediate postoperative period represents a life-threatening complication. Successful treatment with papaverine injected intraluminally by angiographic catheterization has not previously been reported. We present such a case.

\footnotetext{
From Sir Charles Gairdner Hospital (SCGH, Perth, Australia), Department of Cardiothoracic Surgery, Perth, Australia.

Received for publication March 21, 2005; accepted for publication April 5, 2005.

Address for reprints: Lucas H. A. Sanders FCS(SA), MD, Sir Charles Gairdner Hospital (SCGH, Perth, Australia), Department of Cardiothoracic Surgery, Hospital Ave, Nedlands, Perth, WA6007, Australia (E-mail: lucmedi@hotmail.com).

J Thorac Cardiovasc Surg 2005;130:938-40

$0022-5223 / \$ 30.00$

Copyright $\odot 2005$ by The American Association for Thoracic Surgery

doi:10.1016/j.jtcvs.2005.04.022
}

\section{Clinical Summary}

An 84-year-old woman underwent sequential grafting of the first diagonal and left anterior descending coronary artery (LAD) with a pedicled, nonskeletonized left internal thoracic artery (LITA). The procedure was performed without the aid of cardiopulmonary bypass with an Octopus IV Tissue Stabilizer (Medtronic, Inc, Minneapolis, Minn). Preoperative angiography revealed sequential $50 \%$ and $70 \% \mathrm{LAD}$ and $90 \%$ first diagonal stenoses. The right coronary artery was blocked with a small distal vessel to an inferior infarct, well collateralized from the left. Left ventricular function was mildly impaired because of inferior akinesia. Interestingly, left ventriculography revealed hypercontractility in its midportion, resulting in a double-chamber-like image. The patient's history included hypertension, hypercholesterolemia, and peptic ulcer disease. She had stopped smoking 10 years previously. Medications included atenolol, pantoprazole, isosorbide mononitrate, indapamide, ramipril, lercanidipine, pravastatin, nicorandil and clopidogrel (discontinued 3 days before the operation). 